\title{
Hybrid Compound Block Copolymer Micelles Encapsulating Gold Nanoparticles ${ }^{\mathrm{a}}$
}

\author{
Christos Mantzaridis, Stergios Pispas*
}

We report here on the formation of hybrid compound block copolymer micelles encapsulating gold nanoparticles, utilizing a direct and general preparation method. The giant hybrid compound micelles are structured with micelles of PS- $b$-P2VP with gold nanoparticles in their P2VP core and PI-b-PS chains as the outer part of the compound micelles. The gold nanoparticles were produced using gold ion-loaded PS- $b$-P2VP micelles as a nanoreactor, in a PS selective solvent (toluene), by the subsequent reduction of gold ions. The synthesis of the gold nanoparticles was monitored by UV-vis spectroscopy. The gold containing micelles were then encapsulated in larger micelles of PI- $b$-PS copolymer, by successive utilization of toluene and heptane with the intermediate evaporation of toluene. The nanoassembly of the compound materials comprised a PI corona and a PS compound core, with $\mathrm{P} 2 \mathrm{VP} / \mathrm{Au}^{0}$ domains, and was characterized using UV-vis spectroscopy, dynamic light scattering and transmission electron microscopy.
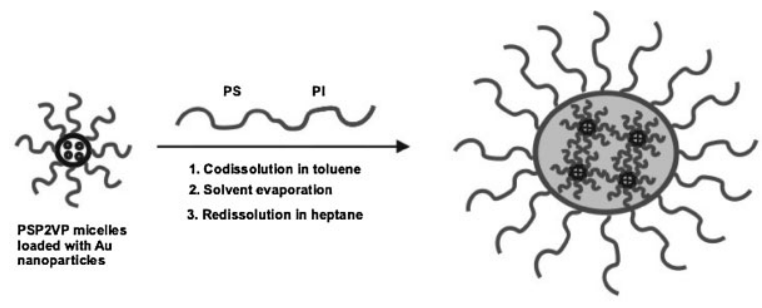

Giant hybrid compound block copolymer

\section{Introduction}

The nanoscale has attracted great attention in recent years. In particular, metal nanoparticles, such as those derived from $\mathrm{Au}, \mathrm{Ni}$, and $\mathrm{Ag}$, exhibit totally different behaviour compared to the bulk material. Their optical properties are completely different when their size is in the nanometer range. This extraordinary optical property has its origins in the surface plasmon resonance phenomena, due to collective oscillations of electrons that are in the conductive band of the metal. These new materials which have unique properties due to their size are called nano-

C. Mantzaridis, S. Pispas

Theoretical and Physical Chemistry Institute, National Hellenic Research Foundation, 48 Vass. Constantinou Ave., 11635 Athens, Greece

Fax: +30210-7273794; E-mail: pispas@eie.gr

${ }^{a}$ 品 Supporting information for this article is available at the bottom of the article's abstract page, which can be accessed from the journal's homepage at http://www.mrc-journal.de, or from the author. particles. ${ }^{[1]}$ The size dependent magnetic, optical and electric properties makes nanoparticles successful candidates for various applications, covering a wide range that varies from optoelectronic and fluorescence sensing devices to medicinal, diagnostic and therapeutic applications. $^{[2]}$

Additionally, the size within the nanoscale and shape of the nanoparticles play a key role in their optical behavior, as previously reported, and various ways of producing different nanoparticles have appeared in the literature. ${ }^{[3]}$ One of the most common and effective methods for the formation of nanoparticles is through utilization of a polymeric nanostructured matrix, which is used as a nanoreactor. These matrices are used to engulf the ionic metal precursors, such as gold, through a variety of interactions. For example, micelles of a diblock copolymer in a selective solvent can encapsulate the precursor ions inside the micellar core or corona. Subsequently a reducing agent is utilized and the ions are reduced to nanoparticles. Various monomeric residues (as a block of diblock copolymers) have been utilized as complexing agents for the metal ions, such as 2- or 4-vinylpyridine, ethylene 
oxide, methacrylic acid etc. ${ }^{[4]}$ The core secured ions are reduced to sizes similar to or smaller than the size of the core. The reduction of the ions can be done via several routes, either with a reduction agent $\left(\mathrm{LiAlH}_{4}\right.$, hydrazine) or with irradiation of the sample. If the core of the micelles is on the nanometer scale, the resulting particles should also be on that scale. Also, the polymeric part does not allow the freshly created nanoparticles to aggregate and precipitate. This experimental technique enables the production of tailormade nanoparticles in terms of the size, and it is quite simple and effective. Recently, some composite materials that contain two different diblock copolymers and gold nanoparticles have been reported in the literature. ${ }^{[5]}$ However, extension of the current synthetic protocols and available nanostructured nanoparticle/ polymer based hybrid materials is necessary in order to facilitate application oriented research.

In the present work, we report on the development of a new nanostructured hybrid polymeric colloidal system, i.e., block copolymer hybrid compound micelles encapsulating metal nanoparticles. The preparation scheme involves the synthesis of gold nanoparticles inside the micelles of a poly(styrene-b-2-vinyl pyridine) (PS-P2VP) diblock copolymer in toluene and the encapsulation of the resulting hybrid micelles in larger micelles of another diblock copolymer, namely poly(isoprene-b-styrene) (PIPS). The giant compound micelles produced in this way were studied in solution and in the dry state by dynamic light scattering (DLS), UV-vis spectroscopy and transmission electron microscopy (TEM).

\section{Experimental Part}

The synthesis of both diblock copolymers was performed by anionic polymerization high vacuum techniques, in order to ensure control over the molecular weight and composition, and narrow polydispersity of the samples. ${ }^{[6]}$ The molecular characteristics of the copolymers were determined by a combination of size exclusion chromatography (SEC) and ${ }^{1} \mathrm{H}$ NMR spectroscopy and are given in Table 1.

\section{Formation of Nanoparticles}

The diblock copolymer PS-P2VP was dissolved in toluene $(c=$ $\left.1 \times 10^{-3} \mathrm{~g} \cdot \mathrm{mL}^{-1}\right)$. In this particular solvent, a selective one for the
PS block, micelles were created with the core formed of P2VP and the corona formed of PS, as evidenced by dynamic light scattering. The solution was equally divided into four vials ( $5 \mathrm{~mL}$ each) and in each of these different quantities of $\mathrm{HAuCl}_{4}$ in ethanol were added, so the resulting four solutions had different gold concentrations, which were $1.25 \times 10^{-8}$ (solution A), $2.5 \times 10^{-8}$ (solution B), $3.75 \times 10^{-8}$ (solution C) and $5 \times 10^{-8} \mathrm{~g} \cdot \mathrm{mL}^{-1}$ solution (solution D).

The solutions were left for $24 \mathrm{~h}$ in order for the $\mathrm{Au}$ ions to be complexed inside the core of the micelles, through interactions with the pyridine group of the P2VP blocks. Subsequently, hydrazine (Alfa Aesar $98 \%+$ ) was added to each solution to reduce the gold ions to metallic gold. After some minutes, all four solutions were coloured from pink to red, depending on the concentration of gold. ${ }^{[7]}$

\section{Preparation of the Hybrid Compound Micelles}

Two series of hybrid compound micelles were prepared in order to ensure that the preparation procedure did not affect the final micelles. In the first series, PI-PS diblock copolymer was dissolved in toluene $\left(c=1 \times 10^{-2} \mathrm{~g} \cdot \mathrm{mL}^{-1}\right)$, which is a good solvent for both PS and PI. The PS-P2VP/Au (solutions A-D) was mixed with $5 \mathrm{~mL}$ of the PI-PS diblock copolymer solution in vials. The solvent was then evaporated and a thin film was created in each vial. Afterwards, $n$ heptane $(10 \mathrm{~mL})$ was added into the vials containing the thin films of the diblocks and $\mathrm{Au}^{0}$ nanoparticles. $n$-Heptane is a selective solvent for the PI block, and a bad solvent for the PS and the P2VP blocks, so micelles of PI-PS with a PI corona and a PS block are favoured in this solvent. The solution was sonicated for $10 \mathrm{~s}$, in a Sonica 3300ETH ultrasonic bath, in order to facilitate the dissolution of the film. The solutions were left in an oven at $60^{\circ} \mathrm{C}$ for $24 \mathrm{~h}$ in order to further facilitate dissolution of the material. After $24 \mathrm{~h}$, the solutions were homogeneous and they had a bluish tint. The general scheme that depicts the formation of the hybrid compound micelles is shown in Scheme 1. All the solutions had the same weight ratio of PI-PS/ PS-P2VP (it was 10:1 while the molar ratio was 158:1) and different gold concentrations for each solution, i.e., $1.25 \times 10^{-8}$ (solution I), $2.5 \times 10^{-8}$ (solution II), $3.75 \times$ $10^{-8}$ (solution III) and $5 \times 10^{-8} \mathrm{~g} \cdot \mathrm{mL}^{-1}$ solution (solution IV).

The second series of solutions were prepared after mixing a certain amount of PS-P2VP/Au ${ }^{0}$ micelles (PS-P2VP micelles containing gold nanoparticles stock solution with $c=1 \times$ $10^{-2} \mathrm{~g} \cdot \mathrm{mL}^{-1}$ polymer and an $\mathrm{Au}$ concentration of $2.5 \times 10^{-8}$ $\mathrm{g} \cdot \mathrm{mL}^{-1}$, by the same way described before) with different amounts of the PI-PS diblock (stock solution with $c=1 \times 10^{-2}$ $\left.\mathrm{g} \cdot \mathrm{mL}^{-1}\right)$. The preparation protocol was analogous to the one described earlier. Solutions were prepared with the same amount of the PS-P2VP/Au ${ }^{0}$ stock solution $(50 \mu \mathrm{L})$ and each time a different

Table 1. Molecular characteristics of the block copolymers utilized in this study.

\begin{tabular}{lccr}
\hline Block copolymer & $\overline{\mathbf{M}}_{\mathbf{w}}$ (by SEC/NMR) & $\overline{\boldsymbol{M}}_{\mathbf{w}} / \overline{\mathbf{M}}_{\mathbf{n}}$ (by SEC) & Composition (by ${ }^{\mathbf{1} H}$ NMR) \\
\cline { 2 - 3 } & $\left(\times 1 \mathbf{0}^{-4}\right)$ & & (wt.-\% PS block) \\
\hline PS-P2VP & 2.7 & 1.05 & 80 \\
PI-PS & 42.4 & 1.04 & 25
\end{tabular}


(2) PSP2VP micelles in toluene

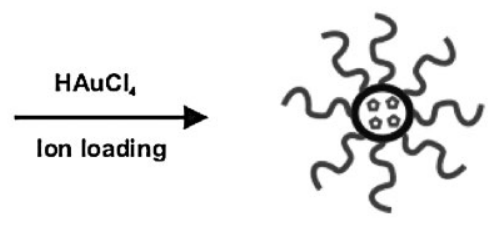

PSP2VP micelles loaded with Au ions

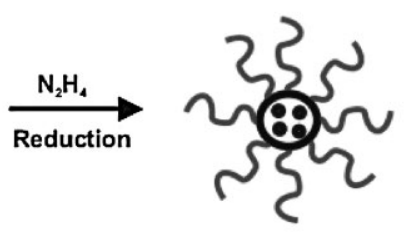

PSP2VP micelles loaded with $\mathrm{Au}$ nanoparticles

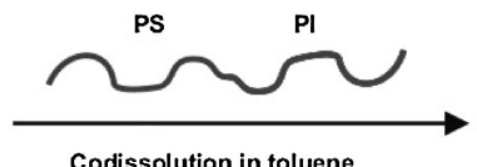

Codissolution in toluene
Solvent evaporation
Redissolution

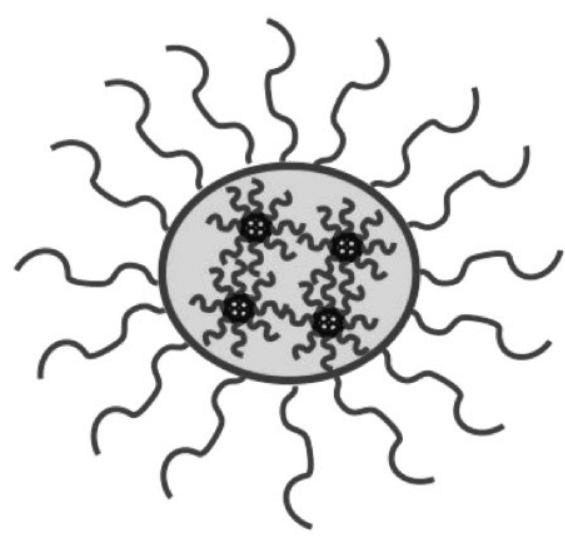

Giant hybrid compound block copolymer micelles in heptane

Scheme 1. General scheme for the formation of the hybrid compound micelles.

amount of the PI-PS stock solution ( $50 \mu \mathrm{L}, 100 \mu \mathrm{L}$, up to $1 \mathrm{~mL}$ ) was added. After the toluene was evaporated, $n$-heptane was added as a solvent. The final weight ratio of PS-P2VP $/ \mathrm{Au}^{0}$ :PI-PS was between 1:1 and 1:20 for the different solutions (molar ratio: 16:1 to 1:1.3), while the final Au concentration was $1.28 \times 10^{-8}$ $\mathrm{g} \cdot \mathrm{mL}^{-1}$. For simplicity, from now on we will refer to these solutions as compound 11, compound 12 , up to compound 120 , respectively.

The formation of gold nanoparticles was confirmed via UV-vis spectroscopy using a Perkin Elmer UV-vis spectrophotometer (Lamda 19). Also, dynamic light scattering experiments were performed in the angular range of 20 to $150^{\circ}$ by a ALV/CGS-3 Compact Goniometer System (ALV GmbH, Germany), using a JDS Uniphase $22 \mathrm{~mW}$ He-Ne laser, operating at $632.8 \mathrm{~nm}$, interfaced with a ALV-5000/EPP multi-tau digital correlator with 288 channels and a ALV/LSE-5003 light scattering electronics unit for stepper motor drive and limit switch control. Autocorrelation functions were measured at least five times and analyzed by the cumulants method and the CONTIN routine. Polydispersities were evaluated from cumulants analysis, through the second cumulant, and are given as values of the ratio $\mu_{2} / \Gamma^{2}$, where $\mu_{2}$ is the second cumulant and $\Gamma$ is the decay rate of the correlation function. Apparent hydrodynamic radii, $R_{\mathrm{h}}$, at different polymer concentrations were calculated using the Stokes-Einstein equation, $R_{\mathrm{h}}=\mathrm{k} T / 6 \pi \eta_{0} D_{\text {app}}$, where $\mathrm{k}$ is the Boltzmann constant, $T$ is the absolute temperature, $\eta_{0}$ is the solvent viscosity and $D_{\text {app }}$ is the diffusion coefficient calculated from the analysis of the correlation function at that particular polymer concentration. Solutions were filtered through $0.45 \mu \mathrm{m}$ PTFE filters before light scattering measurements.

Images from the samples were obtained by using a transmission electron microscopy (TEM) instrument (a JEOL model JEM100C electron microscope) at an accelerating voltage of $80 \mathrm{kV}$ under bright field conditions. Samples were prepared by depositing one drop of the solution onto carbon coated EM copper grids. After $5 \mathrm{~min}$, any excess solvent ( $n$-heptane) was blotted away with a strip of filter paper. No staining was necessary due to the large mass contrast between the polymeric matrices and the gold nanoparticles.

\section{Results and Discussion}

In Figure 1 the UV-vis spectra of four different solutions that contain the compound hybrid micelles and the initial solution of PS-P2VP micelles with gold nanoparticles are depicted. In the spectrum corresponding to the PS-P2VP/Au ${ }^{0}$ system, a rather broad plasmon resonance band is obvious, which indicates nanoparticle formation. The peak of the band is at ca. $664 \mathrm{~nm}$ and, from the unimodality of the band, the nanoparticles seem to be spherical. Based on the

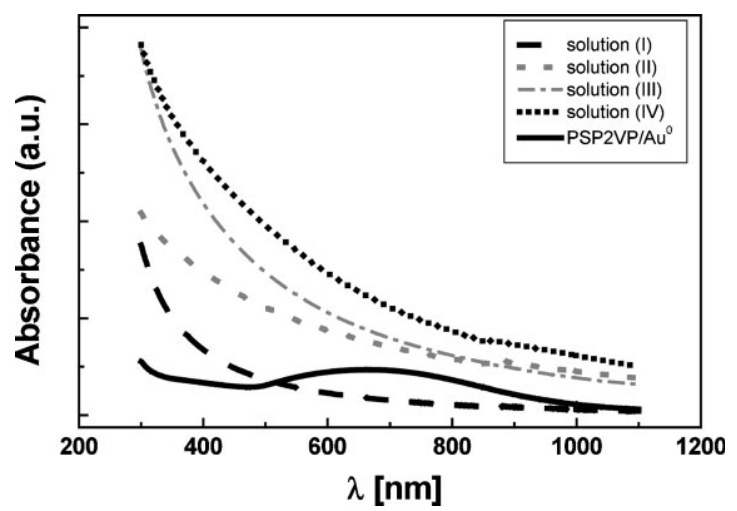

Figure 1. UV-vis spectra of the $\mathrm{PI}-\mathrm{PS} / \mathrm{Ps}-\mathrm{P}_{2} \mathrm{VP} / \mathrm{Au}^{\circ}$ hybrid compound micelles (solutions I-IV) and the initial PS-P2VP micelles with gold nanoparticles (solution concentrations for solutions I-IV are reported in the Experimental Part). 
width of the peak, the particles are expected to be polydisperse and this may be due to the fact that toluene is not a strictly selective solvent for P2VP, leading to not such a confined growth of the particles within the micellar core.$^{[4,7]}$ Using an empirical equation, ${ }^{[8]}$ the diameter of the nanoparticles was calculated to be in the range 5-10 nm. Also taking into account the respective TEM images (see Supporting Information), it can be concluded that a single nanoparticle is formed per PS-P2VP micelle.

From the spectra, it is obvious that some kind of quenching of the plasmon resonance band takes place in the compound hybrid micelles, since no distinct peak is visible. The distinctive plasmon resonance band is replaced by a sloping background absorbance for each of the resulting hybrid compound micelle solutions. A trend of increasing absorbance is observed from the compound hybrid micelles solutions from the lower concentration to the one with the highest concentration of gold nanoparticles. The absence of the distinct plasmon peak may be related to the different environment around the nanoparticles, and the different particle-particle interactions and interparticle distances in the hybrid compound micelle case. ${ }^{[9]}$ The block copolymers do not present any absorption peaks in this wavelength range.

Dynamic light scattering measurements give a more clear view of the changes taking place in the colloidal species in the solutions, in the presence of the PI-PS copolymer. In Figure 2(A), the hydrodynamic radii for each of the hybrid micelles (solutions I-IV) and for the PI-PS micelles in $n$-heptane are shown. It is evident that a major difference in hydrodynamic radii exists, between the empty micelles of PI-PS and those that are loaded with the PS-P2VP $/ \mathrm{Au}^{0}$ micelles. The hydrodynamic radii of the hybrid compound micelles seem to be independent of the final gold concentration (Figure 2(A)), in the concentration range studied. In Figure 2(B), the size distributions for the hybrid compound micelles are shown for solution (II) (see Experimental Part). The hydrodynamic radii distribution of the PS-P2VP and PI-PS micelles are also depicted. The distribution for the PI-PS/PS-P2VP/Au seems to be bimodal; a shoulder at low $R_{\mathrm{h}}$ values exists. Comparison of the distributions of the different micelles shows that the fast mode (lower $R_{\mathrm{h}}$ shoulder) could be attributed to PI-PS micelles that are not loaded with PS-P2VP/Au micelles (the concentration of PI-PS in the solution is significantly higher than that of the PS-P2VP/Au micelles). The slow mode (higher $R_{\mathrm{h}}$ ) should be attributed to the hybrid compound micelles, i.e., PI-PS micelles encapsulating PS$\mathrm{P} 2 \mathrm{VP} / \mathrm{Au}^{0}$ ones. The situation is similar for I-IV solutions (the intensity of the shoulder changes slightly). This is attributed to the fact that a large weight excess of PI-PS was used in this solution series.

Additional experiments with a fixed concentration of PS-P2VP and varying concentrations of PI-PS were made, as
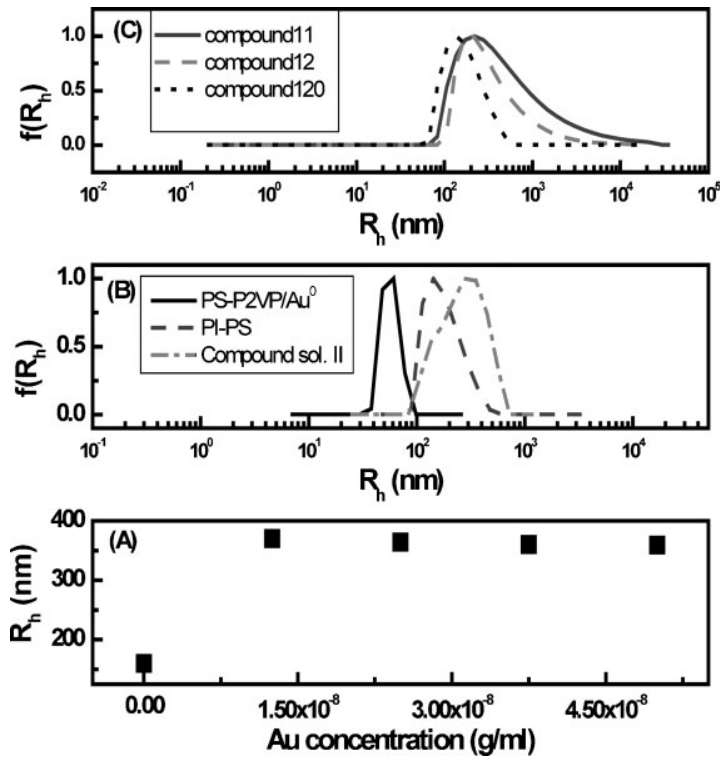

Figure 2. (A) Hydrodynamic radii of the hybrid compound micelles versus $\mathrm{Au}^{\circ}$ concentration (solutions I-IV); (B) Size distribution of hybrid compound micelles (solution II) in comparison to PS-P2VPI $\mathrm{Au}^{\circ}$ and PI-PS micelles; (C) Size distribution of giant hybrid compound micelles at different $\mathrm{PS}-\mathrm{P} 2 \mathrm{VP} / \mathrm{Au}^{\circ}: \mathrm{PI}-\mathrm{PS}$ ratios (solutions of compound 11, compound 12 and compound 120).

described in the Experimental Part. Representative results for compound 11, compound 12 and compound 120 solutions are shown in Figure 2G. The hydrodynamic radii distribution function seems to depend on the concentration of PI-PS. At similar weight ratios of the two components (e.g., compound 11) the resulting hybrid micellar system seems to be rather polydisperse, and as the PI-PS concentration increases (e.g., compound 12, compound 120) the polydispersity of the system decreases. This can be attributed to the fact that, at lower PI-PS concentrations, the PI-PS micelles are encapsulating a number of smaller PS-P2VP/Au micelles (but not all), and this increases the polydispersity of the system. As the number of the PI-PS micelles increases toward an excess over the PS-P2VP/Au micelles, the resulting hybrid compound micelles become less polydisperse, as all of the PS-P2VP/Au micelles are now incorporated into them (this can be monitored by the decrease in the value of the $\mu_{2} / \Gamma^{2}$ parameter, from 0.47 to 0.17 ). This assumption is corroborated by an observation from the previous experiments (solution I-IV), which showed that a certain amount of smaller micelles are encapsulated in larger micelles of PI-PS, and PI-PS micelles in excess can be empty.

In order to gain more information on the structure of the hybrid compound micelles, some TEM images were obtained from the prepared solutions. In Figure 3(A) and 3(B), images from solutions I and II are depicted (see also Figure 1). Spherical particles can be observed in these images. The dark central area should correspond to the 

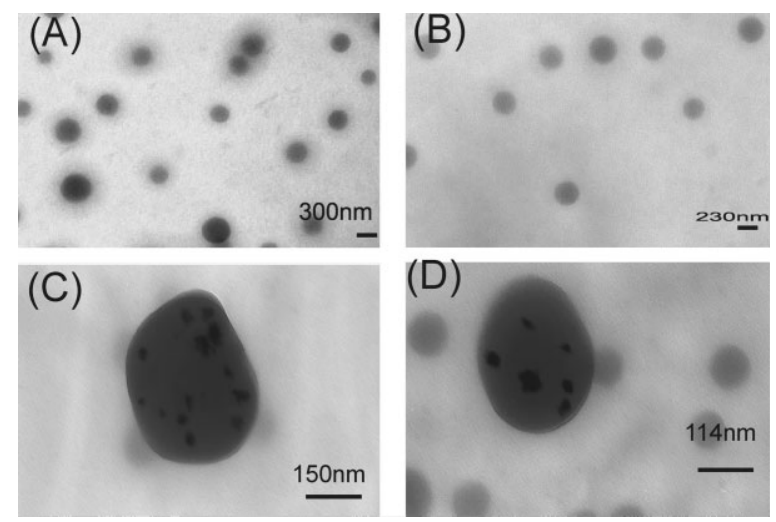

Figure 3. (A), (C): TEM images from hybrid compound micelle solution (I); (B), (D): TEM images from hybrid compound micelle solution (II).

micellar core of the hybrid compound micelles, where the nanoparticles reside, contributing to the higher contrast of the domain. However, the corona of the compound materials is shown as a less dark area around the core. These images denote the spherical core-shell structure of the hybrid compound micelles and the presence of metal nanoparticles in their cores. Also, in Figure 3(C) and (D), higher resolution images are shown for the same solutions. In these images, the core of the compound materials is visible. Darker areas within the cores can be seen, which can be identified as the $\mathrm{Au}^{0}$ nanoparticles. Some aggregation of the nanoparticles within the cores is observed. The random distribution of $\mathrm{Au}^{0}$ nanoparticles should denote the existence of several smaller PSP2VP $/ \mathrm{Au}^{0}$ micelles that exist inside bigger PI-PS micelles. Note also the rather distorted spherical shape of the cores and their size distribution, which may be a result of the encapsulation process using the described experimental protocol. These TEM images directly demonstrate that giant hybrid compound micelles, with encapsulated nanoparticles, have been formed in the mixed copolymer solutions.

\section{Conclusion}

A direct and general method was presented for the preparation of giant compound micelles encapsulating gold nanoparticles. These materials are composed of PI-PS micelles, having within their cores smaller micelles of PSP2VP that contain gold nanoparticles. Encapsulation is facilitated by the compatibility of the PS corona of the PS$\mathrm{P} 2 \mathrm{VP} / \mathrm{Au}^{0}$ micelles with the PS core of the PI-PS micelles. The overall experimental data suggests that these hybrid compound micelles are spherical. This approach for hybrid material preparation could be useful in circumstances that require different outer coronas of the micelles, rather than the one that is used in the nanoreactor scheme for metal nanoparticle production. Needless to say, sub-monolayers, monolayers and films can be produced from the solutions of the hybrid compound micelles, giving rise to nanostructured surfaces and solid nanocomposite hybrid materials. The presented methodology allows for better blending of the hybrid materials with a range of polymeric matrices, when suitably chosen block copolymers and solvents are used.

Acknowledgements: Financial support of this work through the PENED 03ED805 project, supervised by the General Secretariat for Research and Technology/Ministry of Development, Greece, is gratefully acknowledged. The help of Ms. D. Achilleos and Prof. M. Vamvakaki with TEM measurements is also appreciated.

Received: June 30, 2008; Revised: September 8, 2008; Accepted: September 9, 2008; DOI: 10.1002/marc.200800402

Keywords: block copolymers; micelles; nanocomposites; nanoparticles; self-assembly

[1] S. Eustis, M. A. El-Sayed, Chem. Soc. Rev. 2006, 35, 209.

[2] [2a] T. Hasobe, H. Imahori, P. V. Kamat, T. K. Ahn, S. K. Kim, D. Kim, A. Fujimoto, T. Hirakawa, S. Fukuzumi, J. Am. Chem. Soc. 2005, 127, 1216; [2b] H. Imahori, A. Fujimoto, S. Kang, H. Hotta, K. Yoshiada, T. Umeyama, Y. Matano, S. Isoda, Adv. Mater. 2005, 17, 1727; [2c] S. Y. Lin, S. H. Wu, C. H. Chen, Angew. Chem. 2006, 45, 4948; [2d] B. I. Ipe, K. Yoosaf, K. G. Thomas, J. Am. Chem. Soc 2006, 128, 1907; [2e] L. A. Dykman, V. A. Bogatyrev, Russ. Chem. Rev. 2007, 76, 181.

[3] M.-C. Daniel, D. Astruc, Chem. Rev. 2004, 104, 293.

[4] [4a] M. Antonietti, S. Forster, Adv. Mater. 1998, 10, 195; [4b] J. P. Spatz, A. Roescher, M. Moller, Adv. Mater. 1996, 8 , 337; [4c] J. P. Spatz, T. Herzog, S. Mossmer, P. Ziemann, M. Moller, Adv. Mater. 1999, 11, 149; [4d] E. R. Zubarev, J. Xu, A. Sayyad, J. D. Gibson, J. Am. Chem. Soc. 2006, 128, 4958; [4e] G. Hou, D. Chen, M. Jing, Macromolecules 2007, 40, 2134; [4f] A. Meristoudi, S. Pispas, N. Vainos, J. Polym. Sci., Part B: Polym. Phys. 2008, 46, 1515.

[5] X. Chen, Y. Liu, Y. An, J. Lü, J. Li, D. Xiong, L. Shi, Macromol. Rapid Commun. 2007, 28, 1350.

[6] [6a] N. Hadjichrisitidis, H. Iatrou, S. Pispas, M. Pitsikalis, J. Polym. Sci., Part A: Polym. Chem. 2000, 38, 3211; [6b] D. Uhrig, J. W. Mays, J. Polym. Sci., Part A: Polym. Chem. 2005, 43, 6179.

[7] S. Mossmer, J. P. Spatz, M. Moller, T. Aberle, J. Schmidt, W. Burchard, Macromolecules 2000, 33, 4791.

[8] J. Liu, P. Raveendran, Z. Shervani, Y. Ikushima, Y. Hakuta, Chem. Eur. J. 2005, 11, 1854.

[9] X. Liu, X. He, T. Jiu, M. Yuan, J. Xu, J. LV, H. Liu, Y. Li, Chem PhysChem 2007, 8, 906, and references therein. 